\title{
Abnormal Wnt signaling and overexpression of ABCG2 contributes to drug efflux properties of side population cells in nasopharyngeal carcinoma
}

\author{
GUO-FANG GUAN $^{1}$, DE-JUN ZHANG ${ }^{1}$, YING ZHENG ${ }^{2}$, LIAN-JI WEN ${ }^{1}$, \\ DUO-JIAO YU ${ }^{1}$, YAN-QING $\mathrm{LU}^{1}$ and YAN ZHAO ${ }^{1}$ \\ ${ }^{1}$ Department of Otolaryngology, Head and Neck Surgery, The Second Hospital of Jilin University, \\ Changchun, Jilin 130041; ${ }^{2}$ Department of Otolaryngology, Head and Neck Surgery, \\ Tumor Hospital of Jilin Province, Changchun, Jilin 130012, P.R. China
}

Received July 2, 2014; Accepted April 24, 2015

DOI: $10.3892 / \mathrm{mmr} .2015 .3935$

\begin{abstract}
The presence of cancer stem cells (CSCs) has major implications in the choice of cancer treatment strategy and is responsible for tumor relapse. CSCs have been isolated and characterized in several types of cancer; however, studies concerning the CSCs from nasopharyngeal carcinoma (NPC) are limited. Thus, the present study was designed to isolate and characterize the cancer stem-like side population (SP) cells from NPC samples. The fluorescence-activated cell sorting (FACS)-based Hoechst 33342 dye exclusion technique identified that $3.9 \%$ of cells from NPC samples were cancer stem-like SP cells. Upon treatment with verapamil (ABC transporter inhibitor), the percentage of SP cells was significantly reduced to $0.7 \%$, which confirms that the $\mathrm{ABC}$ transporter protein exhibits a significant role in drug exclusion. Fluorescence microscopy analysis revealed that the FACS purified SP cells showed increased expression of ABCG2 (ATP transporter protein), Oct-4 and CD44 (stem cell surface protein). Furthermore, these SP cells exhibited increased mRNA expression of ABCG2 and anti-apoptotic factor Bmi-1, which contribute to multi-drug resistance and increased cell survival rate. Notably, the $\mathrm{Wnt} / \beta$-catenin signaling pathways are altered in SP cells. In addition, using reverse transcription-quantitative polymerase chain reaction analysis it was observed that the cells exhibited increased expression of DKK1 and AXIN2. In conclusion, data from the present study clearly demonstrated that the presence of cancer stem-like SP cells from NPC may be responsible for chemotherapeutic drug resistance, tumor recurrence and invasion.
\end{abstract}

Correspondence to: Dr Ying Zheng, Department of Otolaryngology, Head and Neck Surgery, Tumor Hospital of Jilin Province, 1018 Huguang Road, Changchun, Jilin 130012, P.R. China E-mail: zhengying1231@gmail.com

Key words: anticancer therapy, cancer stem cells, drug resistance, side population cells, nasopharyngeal carcinoma

\section{Introduction}

Nasopharyngeal carcinoma (NPC) is the most common type of cancer that originates in the upper region of the pharynx, and commonly occurs in males and females worldwide (1). Despite recent advances in the treatment strategy, treatment failure and tumor relapse render complete eradication of tumors difficult due to the presence of cancer stem cells (CSCs). The recently proposed CSC theory states that the presence of a small sub-population of cancer cells within the tumor, termed CSCs can cause resistance to therapy and tumor recurrence. Current conventional treatment strategies kill the majority of the neoplastic cells; however, the CSCs are not targeted efficiently and thus are responsible for minimal residual disease (MRD). Therefore it is necessary to target and kill CSCs in order to achieve the complete eradication of tumor cells and to provide long-term disease free survival.

Cancer stem cells have been isolated and characterized in several types of solid tumor by two different methods. Firstly using the expression of stem cell surface markers, such as CD133 and CD44 (2) and secondly using the Hoechst 33342 dye exclusion technique (3). A small population of cells exhibited a distinct FACS profile to the side of the predominant population due to a more efficient Hoechst 33342 dye efflux and lower fluorescent intensity signal. These cells are designated as side population (SP) cells and have been recently well-characterized in several types of tumor, such as small cell lung cancer, glioma, prostate cancer, leukemia, neuroblastoma, hepatoma, nasopharyngeal carcinoma, colorectal cancer, thyroid cancer and lung cancer (3-11). These SP cells exhibit characteristic features of CSCs, such as chemotherapy and apoptosis resistance, elevated expression of $\mathrm{ABC}$ transporter proteins, self-renewal capacity, expression of stem cell surface markers, high tumorigenicity and differentiation potential (12-14). Moreover, SP cells were highly invasive and exhibited abnormal activation of Wnt/ $/$-catenin signaling pathways (15). Therefore, these SP cells are considered as enriched CSCs. Hence, isolation and characterization of these SP cells may aid in elucidating the molecular mechanism underlying the tumorigenesis mediated by CSCs in NPC and 
to target the CSCs effectively. Consequently, the current study aimed to isolate and characterize the CSC-like SP cells from NPC samples, using a FACs-based Hoechst 33342 dye exclusion technique.

\section{Materials and methods}

Sample collection and cell culture. NPC samples were collected at the time of surgery according to the ethical approval. Stage IV, infratemporal NPC samples were taken from 15 patients. The cancer tissues were washed extensively in phosphate-buffered saline (PBS) solution containing antibiotics (Sigma-Aldrich, St. Louis, MO, USA) and were incubated overnight in Dulbecco's modified Eagle's medium (DMEM/F12; Gibco-BRL, Carlsbad, CA, USA) containing $500 \mathrm{U} / \mathrm{ml}$ penicillin, $500 \mu \mathrm{g} / \mathrm{ml}$ streptomycin and $1.25 \mu \mathrm{g} / \mathrm{ml}$ amphotericin B (Gibco-BRL). Enzymatic digestion was performed using $1.5 \mathrm{mg} / \mathrm{ml}$ collagenase (Gibco-BRL) and $20 \mu \mathrm{g} / \mathrm{ml}$ hyaluronidase (Sigma-Aldrich) in PBS for $1 \mathrm{~h}$. Cells were cultured in DMEM with $10 \%$ fetal bovine serum (FBS; Sigma-Aldrich), supplemented with antibiotics and maintained in T-75 flasks at $37^{\circ} \mathrm{C}$ in a humidified $5 \% \mathrm{CO}_{2}$ and $95 \%$ air atmosphere. When cells were $90 \%$ confluent, they were removed from the culture flask using Trypsin-EDTA (0.25\%-53 mM EDTA) washed with phosphate-buffered saline (PBS), and suspended in 10\% DMEM. Cells were counted using a hemocytometer (Z359629; Bright-Line, Sigma-Aldrich, St. Louis, MO, USA). The present study was approved by the Ethics Committee of Tumor Hospital of Jilin Province, (Jilin, China).

Labeling with Hoechst 33342 dye. Groups consisted of: Control, cells labeled with Hoechst 33342 dye alone ( $n=9)$; and drug treated, cells treated with verapamil (Sigma-Aldrich) and labeled with Hoechst 33342 dye (n=9; Sigma-Aldrich). Cells were counted by a hemocytometer and $\sim 10^{6}$ cells $/ \mathrm{ml}$ in $10 \%$ DMEM were labeled with Hoechst 33342 stock (sigma)-bisbenzimide $(5 \mu \mathrm{l} / \mathrm{ml})$ either with dye alone or in combination with $0.8 \mu \mathrm{l} / \mathrm{ml}$ verapamil. Furthermore, cells were counter stained with $2 \mu \mathrm{g} / \mathrm{ml}$ propidium iodide (PI ;Sigma-Aldrich). The cells were sorted using a flow cytometer (Attune NxT; Life Technologies, Carlsbad, CA, USA) and the sorted cells were cultured and maintained in DMEM/F-12 supplemented with 10\% FBS. The Hoechst 33342 dye was excited at $355 \mathrm{~nm}$ and its dual-wavelength fluorescence was analyzed with an optical filter (blue, $450 \mathrm{~nm}$ and red, $675 \mathrm{~nm}$; Omega Optical, Brattleboro, VT, USA).

Immunofluorescent staining. The FACS sorted SP and main population (MP) cells were fixed onto glass slides in ice-cold $4 \%$ paraformaldehyde $\left(4^{\circ} \mathrm{C}\right.$ for $\left.10 \mathrm{~min}\right)$, and blocked with $1 \%$ bovine serum albumin (Sigma-Aldrich) for $30 \mathrm{~min}$ at room temperature (RT) to block nonspecific binding of IgG. After washing in PBS, cells were incubated with fluorescein isothiocyanate (FITC)-conjugated mouse anti-human CD44 (cat. no. HPA005785; 1:200; Sigma-Aldrich) and Oct-4 (cat. no. P0082; 1:200; Abcam, Cambridge, MA, USA) at $4^{\circ} \mathrm{C}$ for $30 \mathrm{~min}$ in the dark. After extensive washing in PBS, cell nuclei were counterstained with Hoechst 33342 and viewed under a confocal microscope.
For ABCG2 expression, SP and MP cells were incubated with mouse anti-human ABCG2 antibodies (cat. no. ab95692; 1L200; Abcam) at $4^{\circ} \mathrm{C}$ overnight. After washing in PBS, cells were combined with horseradish peroxidase-conjugated goat anti-mouse IgG (cat. no. an6789; 1;500; Abcam) and incubated for $30 \mathrm{~min}$ at RT. Cells were counterstained with hematoxylin and mounted with glycerol vinyl alcohol aqueous mounting solution (16). Under an optical microscope (BX51M; Olympus, Tokyo, Japan), the red ABCG2-positive cells were observed.

The immunostaining of tumor spheres generated by SP cells was performed as described previously (17). Spheres were fixed onto glass slides in ice-cold $4 \%$ paraformaldehyde $\left(4^{\circ} \mathrm{C}, 10 \mathrm{~min}\right)$, and blocked with normal serum for $30 \mathrm{~min}$. The cells were then incubated with mouse monoclonal anti-Oct-4 and -CD44 (1:200; Chemicon, Tokyo, Japan) overnight. After washing the slides with PBS, they were incubated with FITC-conjugated goat anti-mouse IgG overnight in a dark room. Nuclei were counterstained with 4,6-diamidino-2-phenylindole (DAPI) and viewed under a fluorescence microscope (LSM 510; Carl Zeiss GmbH, Jena, Germany). All images were processed with Adobe Photoshop CS5 (Adobe Systems, Inc., CA, USA).

Cell resistance assay. Approximately $1 \times 10^{3}$ cells/plate were cultured in 96-well plates and treated with $10 \mu \mathrm{g} / \mathrm{ml}$ 5-fluorouracil (5-FU; Sigma-Aldrich), $20 \mu \mathrm{mol} / 1$ cisplatin (Sigma-Aldrich), $2 \mu \mathrm{mol} / 1$ paclitaxel (Sigma-Aldrich) and $2 \mu \mathrm{g} / \mathrm{ml}$ docetaxel (Sigma-Aldrich). The mean value of optical density $(\mathrm{OD})_{450}$ obtained was presented as a graph. Cell resistance in the two groups was calculated using the following formula: Cell resistance rate $(\%)=\left(\right.$ experimental group $\mathrm{OD}_{450}$ value/control group $\mathrm{OD}_{450}$ value) x 100 , as described previously (18).

Reverse transcription (RT)-quantitative polymerase chain reaction $(P C R)$. Total RNA was extracted and complementary DNA was prepared using a reverse transcriptase kit (Fermentas, Vilnius, Lithuania). First-strand cDNA synthesis and qPCR were performed using the PrimeScript RT-PCR kit (Takara, Otsu, Japan) and the SYBR Premix Ex Taq II kit (Takara, Otsu, Japan), according to the manufacturer's instructions. RT-qPCR analysis was subsequently performed on an iCycler IQ real-time detection system (Bio-Rad, Hercules, CA, USA), using IQ Supermix with SYBR-Green (Bio-Rad). The sequences of human specific primers used were as follows: Forward: CTGGCTTTGGTGAACTGTTG and reverse: AGTTGCTCACAGCCAAGACA for AXIN2; forward: AGCACCTTGGATGGGTATTC and reverse: CACAATCCTGAGGCACAGTCDKK1 for DKK1; forward: TCAATCAAAGTGCTTCTTTTTTATG and reverse: TTGTGGAAGAATCACGTGGCABCG2 for ABCG2; forward: ATGTCGTGGAGTCTACTGGC and reverse: TGACCTTGCCCACAGCCTTG for GAPDH; and forward: CTCCCAACTGGTTCGACCTT and reverse: CGGTTTCCATATTTCTCAGT for BMI-1. The amplified products were separated by electrophoresis on ethidium bromide-stained $1.2 \%$ agarose gels. Band intensity was measured by Image $\mathrm{J}$ from two independent experiments.

Biochemistry. For western blot analysis, proteins were extracted from the SP and MP cells, and protein concentration 

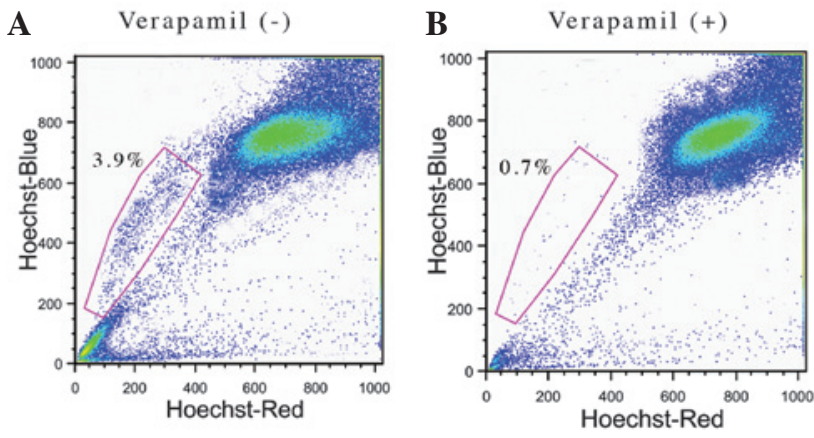

Figure 1. A representative image of dot plot analysis of fluorescence-activated cell sorting. (A) Graph showing 3.9\% of total cells from NPC are SP cells in the gated region, which efflux Hoechst 33342 dye out of the cell. (B) The verapamil-treated NPC cells exhibited a significantly reduced percentage of SP cells $(0.7 \%)$ in the gated region. NPC, nasopharygeal carcinoma; SP, side population.
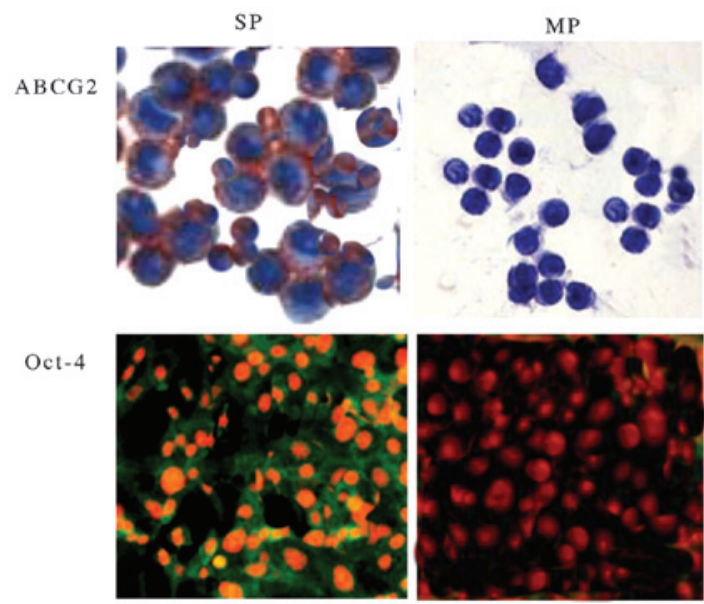

CD 44
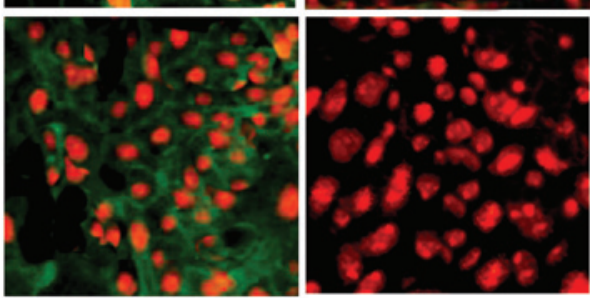

Figure 2. Immunocytochemistry of the sorted NPC SP and MP cells. SP cells showing enhanced expression of ABCG2 (red colour) and increased expression of stem cell surface markers CD133 and Oct-4 when compared with MP cells (magnification, x100). NPC, nasopharyngeal carcinoma; SP, side population; MP, main population.

was determined using the Bradford assay (19). Following $10 \%$ sodium dodecyl sulfate-polyacrylamide gel electrophoresis (Bio-Rad, Hercules, CA, USA), proteins were transferred to a nitrocellulose membrane (Bio-Rad), the gels were treated with rabbit anti-human ABCG2, actin and Bcl-2 primary antibodies, goat anti-rabbit IgG with alkaline phosphatase markers secondary antibody and a chemiluminescence reagent. Blots were detected and scanned using a densitometer (Bio-Rad GS-710).

Sphere formation assay. A sphere formation assay was performed exactly as described previously (20). The sorted SP cells and MP were placed at a density of 1,000 cells $/ \mathrm{ml}$,

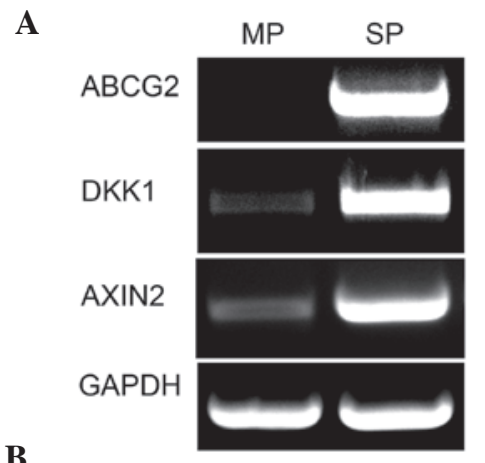

B

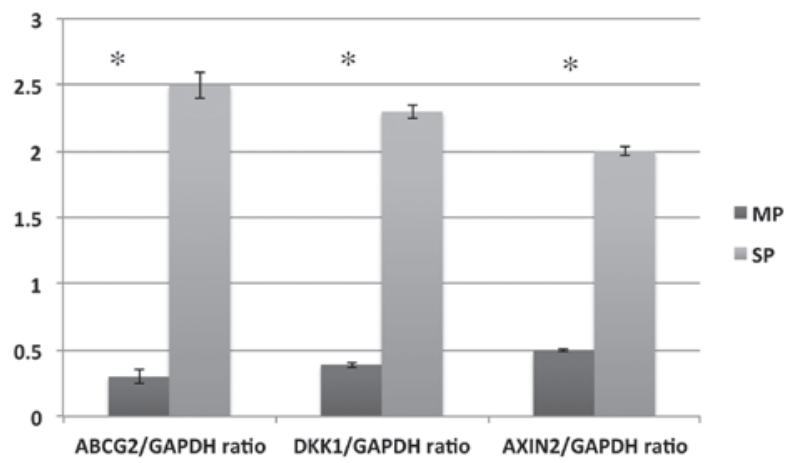

Figure 3. Increased expression of $\mathrm{ABCG} 2$ and abnormal activation of Wnt $/ \beta$-catenin signaling in SP cells. (A) Reverse transcription-quantitative polymerase chain reaction analysis of ABCG2 and endogenous Wnt target genes. The expression of ABCG2, DKK1 and AXIN2 was highly expressed in SP cells compared with MP cells. GAPDH was used as a house keeping gene. (B) Quantification from three separate independent experiments suggest that the expression levels of ABCG2, DKK1 and AXIN2 are significantly higher in SP cells. Error bars represent the standard deviation. ${ }^{* *} \mathrm{P}<0.01$, compared with MP cells.

resuspended in tumor sphere medium consisting of serumfree 1:1 mixture of Ham's F-12/DMEM (Sigma-Aldrich), N2 supplement (Sigma-Aldrich), $10 \mathrm{ng} / \mathrm{ml}$ human recombinant bFGF and $10 \mathrm{ng} / \mathrm{ml}$ EGF (R\&D Systems, Inc., Minneapolis, MN, USA), and subsequently cultured in ultra-low attachment plates for $\sim 2$ weeks. Sorted SP and MP cells were seeded at a low density of 20 cells/1 and the number of generated spheres ( $>100$ cells/ml) was counted after 7 days of culture.

Statistical analysis. One-way analysis of variance and Student's t-test was performed to determine the significant difference between the treatment and control groups. Statistical analysis was performed using SAS statistical software (SAS Institute, Inc., Cary, NC, USA). $\mathrm{P}<0.01$ was considered to indicate a statistically significant difference.

\section{Results}

Analysis of ABCG2 expression and stem cell surface proteins in SP cells. NPC samples were analyzed for the presence of cancer stem-like SP cells by a Hoechst 33342 dye exclusion technique. During FACS analysis, the dead cells were excluded by PI staining and therefore, the live cells were carefully selected. After setting the gate for the ability of the cells to expel the Hoechst 33342 dye, $3.9 \%$ of the total cells were identified to be SP cells (Fig. 1A) and the remaining cells were considered to be MP cells. The Hoechst 33342 exclusion 


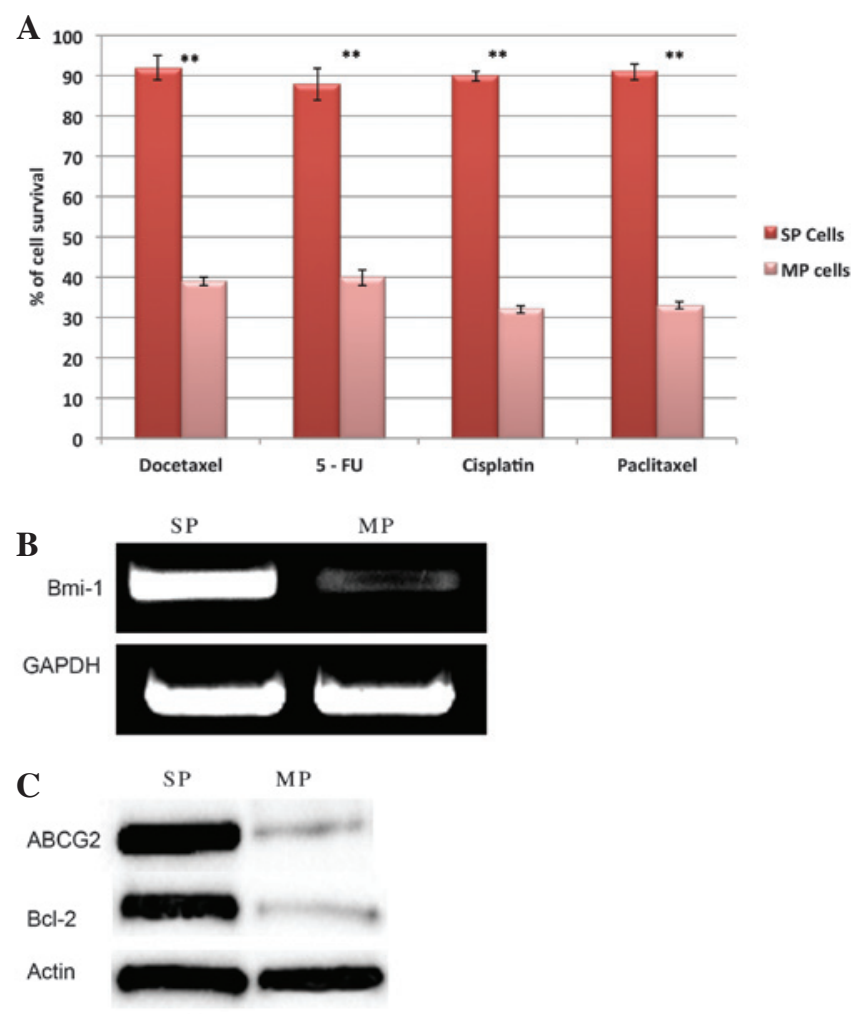

Figure 4. (A) Fluorescence-activated cell sorting showed a significantly increased cell survival rate of SP cells compared with MP cells after treatment with drugs, 5-FU, cisplatin, docetaxel and paclitaxel. The error bar represents the standard deviation. ${ }^{* *} \mathrm{P}<0.01$, compared with MP cells. (B) Reverse transcription-quantitative polymerase chain reaction analysis shows elevated expression of anti-apoptotic gene Bmi-1 in SP cells when compared with MP cells. GAPDH is used as a house keeping gene. (C) Western blot analysis of protein expression levels in SP and MP cells. Equal concentrations of protein were loaded per lane. SP, side population; MP, main population; 5-FU, 5-fluorouracil.

property of SP cells involves the overexpression of multi-drug resistance transporter 1 (MDR1), a member of the ABC transporter transmembrane protein family. Verapamil is a MDR1 transporter protein inhibitor, which efficiently inhibits the drug efflux action by the SP cells. Following verapamil treatment, the percentage of SP cells was significantly reduced to $0.7 \%$ (Fig. 1B). The data confirms that the presence of ABC transporters in SP cells are responsible for chemotherapy resistance.

The SP and MP cells were then analyzed to compare the expression level of ABCB2 genes and other stem cell genes, such as OCT- 4 and CD44 by fluorescence microscopy. As shown in Fig. 2, FACS-sorted SP cells showed increased expression of ABCG2, CD44 and Oct-4 compared with MP cells. The increased expression of CD44 and Oct- 4 in SP cells indicated that SP cells have a high capacity for self-renewal. In addition to this data, elevated mRNA expression of ABCG2 in SP cells was also observed (Fig. 3A). Hence, the data clearly suggest that elevated expression of ABCG2, CD44 and Oct-4 in SP cells may be crucial in drug resistance and tumor relapse of NPC.

Abnormal activation of Wnt $\beta$-catenin signaling and multi-drug resistance of SP cells. The Wnt/ $\beta$-catenin signaling pathway

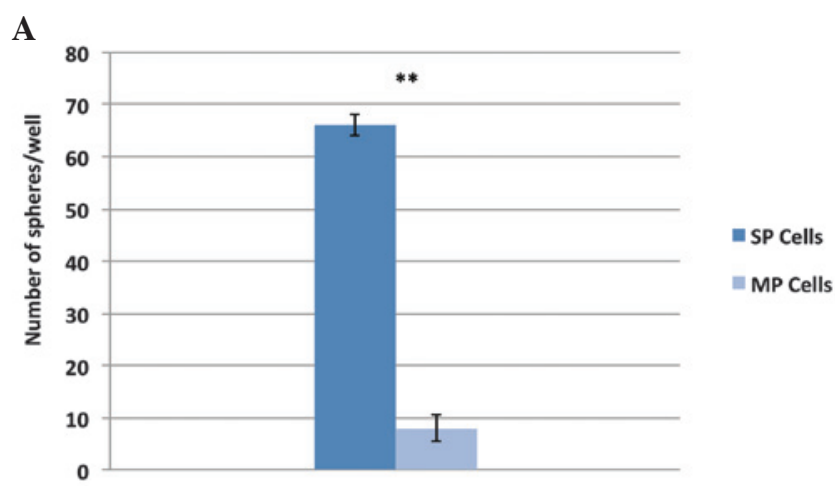

B

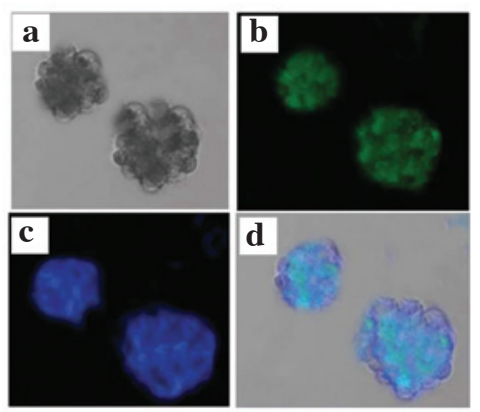

Figure 5. Tumor sphere formation. (A) Clone formation efficiency of NPC SP cells. The total number of tumor spheres generated by NPC SP cells are significantly higher than that of MP cells. The error bar represents the standard deviation." $\mathrm{P}<0.01$, compared with MP cells. (B) The tumor spheres generated by SP cells are positive to CD44 (green). Cell nuclei were counterstained with Hoechst 33342 (blue). (a) Brightfield; (b) CD44; (c) Hoechst; (d) merge.

has been found to be associated with the self-renewal property of cancer cells. Using a TOPFLASH luciferase reporter assay, it was previously reported that activity of $\beta$-catenin-dependent transcription and elevated expression of DKK1 and AXIN2 was found in HNSCC SP cells compared with non-SP cells (21). Similar to these findings, RT-qPCR results also showed that the expression levels of two Wnt/ $\beta$-catenin target genes, DKK1 and AXIN2 are significantly elevated in SP cells compared with MP cells (Fig. 3A and B).

In order to characterize the SP cells, the self-renewal potential and drug resistance of SP cells was analyzed by sphere formation and drug resistance assays. It was observed that SP cells are highly resistant to multiple drugs, such as docetaxel, 5-FU, cisplatin and paclitaxel. The drug-treated SP cells were more resistant and exhibited significantly increased survival rates when compared with MP cells (Fig. 4A). Furthermore, RT-qPCR and western blot analysis data revealed that SP cells exhibit elevated mRNA expression of the anti-apoptotic gene Bmi-1 (Fig. 4B) and increased expression of ABCG2 and Bcl-2 protein (Fig. 4C). ABCG2 and anti-apoptotic factors are crucial factors involved in drug and apoptotic resistance of SP cells. It was also observed that FACS-purified SP cells can generate tumor spheres rapidly and the total number of spheres generated by NPC SP cells are significantly higher than that by MP cells (Fig. 5A). Furthermore, the NPC SP cell generated tumor spheres are immunopositive for $\mathrm{CD} 44$ (Fig. 5B). The data clearly demonstrated that NPC SP cells are responsible for chemotherapeutic resistance and may be involved in rapid tumor recurrence and invasion. 


\section{Discussion}

The presence of CSCs results in the failure of treatment due to the development of MRD. Current conventional treatment strategies efficiently kill the majority of the neoplastic cells and but fail to kill the CSCs. According to the CSC theory, CSCs are the predominant factor in multi-drug resistance. These CSCs are also responsible for tumor growth, metastasis and tumor invasion (22). It was reported that CSCs can survive conventional anticancer therapies owing to the overexpression of efflux pumps, which ultimately contributed to multi-drug resistance (23). Therefore, it is essential to eliminate the CSCs in order to completely eradicate the tumor and prevent MRD.

The proposed method to identify the CSCs is the FACS-based Hoechst 33342-dye exclusion technique in which a small population of cancer cells efflux this dye and are termed SP cells (22). These SP cells exhibit the features of CSCs, such as drug resistance, self-renewal capacity, high differentiation potential, expression of stem cell surface markers and apoptosis resistance. Hence, isolation and further characterization of SP cells aids in the development of novel anticancer drugs, which could efficiently target and kill the CSCs.

In the present study, $3.9 \%$ of the total cells were observed to be SP cells in the NPC sample using the Hoechst 33342 dye exclusion method. Upon treatment with verapamil, the prevalence of SP cells was reduced to $0.7 \%$, which confirms the existence of the $\mathrm{ABC}$ transporter protein. In addition, gene and protein expression studies as well as immunofluorescence clearly showed that $\mathrm{SP}$ cells overexpressed the $\mathrm{ABC}$ transporter protein, ABCG2. Hence, these findings suggest that chemotherapy resistance of SP cells from NPC actively involves the overexpression of ABCG2.

The OCT-4 gene was shown to be involved in cell proliferation (24) and the survival of CSCs is partly mediated through the Oct-4/Tcl1/Akt1 pathway (25). Similarly, the cell surface proteoglycans, such as glycoprotein CD44, are important in tumor invasion and metastasis in several types of tumor (26). Furthermore, the overexpression of CD44 was shown to be involved in chemo- and radioresistance (27). Also, CSCs are highly resistant to death-inducing signals due to the enhanced expression level of anti-apoptotic proteins and high levels of drug transporters $(7,18)$. It was previously reported that NPC samples showed increased expression of CD44 positive cells, which may be responsible for high levels of tumorigenesis and metastasis (2). In line with these findings, it was also demonstrated that NPC SP cells have increased expression of CD44, Oct-4, Bcl-2 and Bmi-1 compared with MP cells. Therefore, there may be significant functional interaction between ABCG2, CD44, Oct-4, Bmi-1 and Bcl-2 in the contribution of chemotherapy/apoptosis resistance, tumorigenesis and invasion of NPC cells. In addition, it was also demonstrated that FACs purified SP cells showed high resistance to the chemotherapeutic agents and have increased cell survival rates compared with MP cells. These results clearly confirm that overexpression of efflux pumps (ABCG2) and increased expression of anti-apoptotic factor Bcl-2 are the predominant factors, which confer the properties of multidrug resistance and treatment failure in NPC. Furthermore, the SP cells exhibit self-renewal capacity as they generate more tumor spheres than non-SP cells. Recently, it was shown that secretion of certain interleukins, such as IL-3 and IL-4, promotes enhanced survival rates by altering the rate of apoptosis (28-30). Therefore, it was also hypothesized that secretion of interleukins in NPC SP cells may be involved in apoptosis resistance.

Notably, it was found that the Wnt/ $/$-catenin pathway is highly activated in NPC SP cells. Abnormal activation of Wnt/ $\beta$ catenin in HNSCC was shown to be involved in chemotherapy resistance and invasive growth of cancer cells (31-33). Similarly, enhanced Wnt/ $\beta$-catenin pathway activation is the phenotype of NPC SP cells and may also contribute to multi-drug resistance and tumor invasion (31). However, the detailed mechanism of altered Wnt/ $\beta$-catenin pathway signaling requires detailed investigation. In conclusion, the data suggest that isolation and characterization of SP cells may provide valuable information to improve cancer treatment and to design a novel anticancer drug that effectively kills CSCs and prevents MRD in NPC.

\section{Acknowledgements}

The authors would like to thank Dr Wanshan Li, Department of Oral and Maxillofacial Surgery, Chongqing Medical University for sharing the FACS protocol by personal communication.

\section{References}

1. Kam MK, Wong FC, Kwong DL, Sze HC and Lee AW: Current controversies in radiotherapy for nasopharyngeal carcinoma (NPC). Oral Oncol 50: 907-912, 2014.

2. Su J, Xu XH, Huang Q, Lu MQ, et al: Identification of cancer stem-like CDZ cells in human nasopharyngeal carcinoma cell line. Arch Med Res 42: 15-21, 2011.

3. Goodell MA, Brose K, Paradis G, Conner AS and Mulligan RC: Isolation and functional properties of murine hematopoietic stem cells that are replicating in vivo. J Exp Med 183: 17971806,1996

4. Salcido CD, Larochelle A, Taylor BJ, Dunbar CE and Varticovski L: Molecular characterisation of side population cells with cancer stem cell-like characteristics in small-cell lung cancer. Br J Cancer 102: 1636-1644, 2010.

5. Yin B, Yang Y, Zhao Z, Zeng Y, Mooney SM, et al: Arachidonate 12-lipoxygenase may serve as a potential marker and therapeutic target for prostate cancer stem cells. Int J Oncol 38: 1041-1046, 2011 .

6. Tabuse M, Ohta S, Ohashi Y, Fukaya R, et al: Functional analysis of HOXD9 in human gliomas and glioma cancer stem cells. Mol Cancer 10: 60, 2011

7. Feuring-Buske M and Hogge DE: Hoechst 33342 efflux identifies a subpopulation of cytogenetically normal CD34(+) CD38(-) progenitor cells from patients with acute myeloid leukemia. Blood 97: 3882-3889, 2001.

8. Hirschmann-Jax C, Foster AE, Wulf GG, Nuchtern JG, et al: A distinct 'side population' of cells with high drug efflux capacity in human tumor cells. Proc Natl Acad Sci USA 101: 1422814233, 2004.

9. Mitsutake N, Iwao A, Nagai K, Namba H, et al: Characterization of side population in thyroid cancer cell lines: cancer stem-like cells are enriched partly but not exclusively. Endocrinology 148: 1797-1803, 2007.

10. Haraguchi N, Utsunomiya T, Inoue H, Tanaka F, Mimori K, Barnard GF and Mori M. Characterization of a side population of cancer cells from human gastrointestinal system. Stem Cells 24: 506-513, 2006.

11. Ho MM, Ng AV, Lam S and Hung JY: Side population in human lung cancer cell lines and tumors is enriched with stem-like cancer cells. Cancer Res 67: 4827-4833, 2007.

12. Bourguignon LY, Peyrollier K, Xia W and Gilad E: Hyaluronan-CD44 interaction activates stem cell marker Nanog, Stat-3-mediated MDR1 gene expression and ankyrinregulated multidrug efflux in breast and ovarian tumor cells J Biol Chem 283: 17635-17651, 2008. 
13. Gao AC, Lou W, Sleeman JP and Isaacs JT: Metastasis suppression by the standard CD44 isoform does not require the binding of prostate cancer cells to hyaluronate. Cancer Res 58: 2350-2352, 1998.

14. Wang J, Guo LP, Chen LZ, Zeng YX and Lu SH: Identification of cancer stem cell-like side population cells in human nasopharyngeal carcinoma cell line. Cancer Res 67: 3716-3724, 2007.

15. Reya $\mathrm{T}$ and Clevers $\mathrm{H}$ : Wnt signaling in stem cells and cancer. Nature 434: 843-850, 2005

16. Zhang H, Liu W, Feng X, Wang L, Jiang X, et al: Identification of ABCG2+ cells in nasopharyngeal carcinoma cells. Oncol Rep 27: 1177-1187, 2012.

17. Ma L, Lai D, Liu T, et al: Cancer stem-like cells can be isolated with drug selection in human ovarian cancer cell line SKOV3. Acta Biochim Biophys Sin 42: 593-602, 2010.

18. He QZ, Luo XZ, Wang K, et al: Isolation and characterization of cancer stem cells from high-grade serous ovarian carcinomas. Cell Physiol Biochem 33: 173-184, 2014.

19. Lowry OH, Rosebrough NJ, Farr AL and Randall RJ: Protein measurement with the Folin phenol reagent. J Biol Chem 193: 265-275, 1951

20. Yanamoto S, Kawasaki G, Yamada S, Yoshitomi I, Kawano $\mathrm{T}$, et al: Isolation and characterization of cancer stem-like side population cells in human oral cancer cells. Oral Oncol 47: 855-860, 2011.

21. Song J, Chang I, Chen Z, Kang M and Wang CY: Characterization of side populations in HNSCC, highly invasive, chemoresistant and abnormal wnt signaling. PLoS One 5: e11456, 2010.

22. Gil J, Stembalska A, Pesz KA and Sasiadek MM: Cancer stem cells: the theory and perspectives in cancer therapy. J Appl Genet 49: 193-199, 2008.

23. Komuro H, Saihara R, Shinya M, Takita J, et al: Identification of side population cells (stem-like cell population) in pediatric solid tumor cell lines. J Pediatr Surg 42: 2040-45, 2007.
24. Campbell PA, Perez-Iratxeta C, Andrade-Navarro MA and Rudnicki MA: Oct4 targets regulatory nodes to modulate stem cell function. PLoS One 2: e553, 2007.

25. Hu T, Liu S, Breiter DR, Wang F, Tang Y and Sun S: Octamer 4 small interfering RNA results in cancer stem cell-like cell apoptosis. Cancer Res 68: 6533-6540, 2008.

26. Ponta H, Sherman L and Herrlich PA. CD44: From adhesion molecules to signalling regulators. Nat Rew Mol Cell Bio 4: 33-45, 2003.

27. Honeth G, Bendahl PO, Ringnér M, Saal LH, Gruvberger-Saal SK, et al: The $\mathrm{CD} 44^{+} / \mathrm{CD} 24^{-}$phenotype is enriched in basal-like breast tumors. Breast Cancer Res 10: R53, 2008.

28. Dancescu M, Rubio-Trujillo M, Biron G, Bron D, Delespesse G and Sarfati M: Interleukin 4 protects chronic lymphocytic leukemic B cells from death by apoptosis and upregulates Bcl-2 expression. J Exp Med 176: 1319-1326, 1992.

29. Kieslinger M, Woldman I, Moriggl R, et al: Antiapoptotic activity of Stat5 required during terminal stages of myeloid differentiation. Genes Dev 14: 232-244, 2000.

30. Prokopchuk O, Liu Y, Henne-Bruns D and Kornmann M: Interleukin-4 enhances proliferation of human pancreatic cancer cells: Evidence for autocrine and paracrine actions. Br J Cancer 92: 921-928, 2005.

31. Li J and Wang CY: TBL1-TBLR1 and beta-catenin recruit each other to Wnt target-gene promoter for transcription activation and oncogenesis. Nat Cell Biol 10: 160-169, 2008.

32. Yang F, Zeng Q, Yu G, Li S and Wang CY: Wnt/beta-catenin signaling inhibits death receptor-mediated apoptosis and promotes invasive growth of HNSCC. Cell Signal 18: 679-687, 2006.

33. Chen S, Guttridge DC, You Z, Zhang Z, Fribley A, et al: Wnt-1 signaling inhibits apoptosis by activating b-catenin/Tcf-mediated transcription. J Cell Biol 152: 87-96, 2011. 